NASZA DERMATOLOGIA Online OUR DERMATOLOGY Online

Source of Support:

Georgia Dermatopathology Associates, Atlanta, Georgia, USA

Competing Interests: None

\section{NEURAL REACTIVITY DETECTED BY IMMUNOFLUORESCENCE IN A PATIENT WITH A LOCALIZED BLISTERING DISEASE}

\author{
Ana Maria Abreu Velez, Michael S. Howard \\ Georgia Dermatopathology Associates, Atlanta, Georgia, USA
}

Corresponding author: Ana Maria Abreu Velez, MD PhD

abreuvelez@yahoo.com

\begin{abstract}
Introduction: Multiple clinical etiologies exist for rapidly appearing skin blisters are multiple.

Case report: A 69-year-old male from a rural area of Georgia, USA, was evaluated for the presence of suddenly appearing, localized erythema and edema on his leg with a skin blister. At presentation, the patient was taking multiple medications for other medical issues.

Methods: Skin biopsies for hematoxylin and eosin (H\&E) examination, as well as for direct immunofluorescence, indirect immunofluorescence and immunohistochemistry studies were performed.

Results: H\&E staining demonstrated a subepidermal blistering process. Within the dermis, a mild, superficial, perivascular and perineural infiltrate of lymphocytes, histiocytes and eosinophils was seen. Direct and indirect immunofluorescence revealed focal dermal perineural and free epidermal nerve fiber staining utilizing antibodies to human Complement/C1q, C3, C4, kappa and lambda light chains. Immunohistochemistry studies revealed $\operatorname{IgG}, \operatorname{IgE}, \mathrm{C} 3$, fibrinogen, albumin and kappa deposition inside and around the blister.

Conclusions: Our case has some clinical and epidemiological features that resemble localized bullous pemphigoid, however, the most interesting findings were the neural reactivity without concomitant diabetes or peripheral neuropathy. Notably, a concomitant leak of ricin was simultaneously detected in same geographic area as the patient. We suggest that ricin environmental toxicity may have contributed to the observed neural reactivity.
\end{abstract}

Key words: intraepidermal nerve fibers; ricin; blistering agents; direct immunofluorescence

Abbreviations and acronyms: Monkey esophagus (ME), direct immunofluorescence (DIF), indirect immunofluorescence (IIF), immunohistochemistry (IHC), basement membrane zone (BMZ), hematoxylin and eosin (H\&E), fluorescein isothiocyanate (FITC), 4',6-diamidino-2-phenylindole (Dapi).

\section{Introduction}

Bullous diseases can be of autoimmune etiology, or caused by medications, diabetes, insect bites, porphyries, and other causes [1-4]. Blistering can be elicited by multiple medications, either prescribed or over-the-counter, as well as natural or synthetic dietary supplements [1-4]. Blisters may be the major clinical feature of the overall disorder; alternatively, blisters may be seen focally in localized areas of a more extensive rash [1-4]. Some of the known blistering diseases may overlap clinically.

\section{Case report}

A 69-year-old male was evaluated for blisters of a few days duration, accompanied by pain and itching. On physical examination, the patient displayed a single, intact tense bulla with mild erythema at its base. A second, adjacent site on the abdomen was consistent with a ruptured bulla with a denuded surface. The patient had a past medical history of multiple non-venomous insect bites on the legs, bronquiectasia, and urticaria. The patient was specifically medicated with bisoprolol fumarate $5 \mathrm{mgs}$ orally daily, ipratropim bromide $0,02 \%$ inhalation solution 3 times at day via nebulization, clonazepam $0,5 \mathrm{mgs}$ one tablet at day, Nexium $^{\mathrm{TM}} 40$ miligrams one tablet a day, Proair HFA $^{\mathrm{TM}}$ (albuterol sulfate; $90 \mathrm{mgs}$ base) nebulization aerosol solution 2 puffs every 4 hours as needed, and Xopenex ${ }^{\mathrm{TM}} 0.63 \mathrm{mg} / 3 \mathrm{ml}$ inhalation nebulization 3 times a day. The patient was living in a rural area and had no clinical history of diabetes or peripheral neuropathy. 
Clinically, the patient was diagnosed with bullous pemphigoid and was treated initially with betamethasone $0.05 \%$ cream, doxycyclin $100 \mathrm{mg}$ a day and niacinamide; no improvement was achieved on this regimen. Next, the dermatologist prescribed a regimen of 1) prednisone $10 \mathrm{mg}$ a day each morning for 3 weeks, increasing to a dosage of 2.5 mg prednisone every second or third day; 2) Os-Cal Extra D3 ${ }^{\mathrm{TM}} 500 \mathrm{UI}$ a day; and 3) hydroxyzine $\mathrm{HCl} 25 \mathrm{mg}$ tablets, 1 or 2 tablets at night for itching. The lesions improved after several weeks of this therapeutic regimen.

\section{Methods}

Skin biopsies for 1) hematoxylin and eosin (H\&E), 2) direct immunofluorescence (DIF), 3) indirect immunofluorescence (IIF) utilizing monkey esophagus (ME) substrate and 4) immunohistochemistry (IHC) stains were performed as previously described [6-9]. An additional sodium chloride salt split skin IIF technique was performed as previously described [4-6]. For the IIF we also used Texas red conjugated armadillo repeat gene deleted in velocardiofacial syndrome (ARVCF) antibody from Progen Germany.

\section{Results}

\section{Microscopic and immunofluorescence findings:}

Examination of the H\&E tissue sections demonstrates a subepidermal blistering disorder (Fig. 1). Within the blister lumen, numerous eosinophils were present, with occasional lymphocytes also seen. Neutrophils were rare. Within the dermis, a florid, superficial, perivascular and perineural infiltrate of lymphocytes, histiocytes and eosinophils was identified. No vasculitis was present. A PAS special stain revealed reinforcement around most dermal nerves, and no fungal organisms. DIF displayed $\operatorname{IgG}(+++$, diffuse dermal perineural); IgA (-); IgM (+++, diffuse dermal perineural); $\operatorname{IgD}(+$, focal dermal perineural); IgE (-); Complement/C1q $(+++$, diffuse dermal perineural); $\mathrm{C} 3(+++$, diffuse dermal perineural); C4 (+, focal dermal perineural); kappa light chains (+, diffuse dermal perineural); lambda light chains $(++$, diffuse dermal perineural); albumin( + , focal dermal perineural) and fibrinogen ( + , focal dermal perineural) (Fig. 1,2). Sodium chloride split skin IIF did not reveal significant dermal/epidermal junctional staining.

\section{Discussion}

We report a senior patient from a rural area who clinically suddenly developed erythematous plaques and focal blisters. Although clinically his diagnosis was considered to be bullous pemphigoid (BP), the DIF and IIF findings were not representative for that diagnosis. A bullous allergic drug reaction was the second differential diagnosis; however, contrary to most allergic drug reactions, the DIF findings of significant fibrinogen deposition around dermal blood vessels was not found [6]. Notably, BP can cause some neural pathologic sequelae [7]. Our immunologic findings indicated that the primary immunopathologic process was directed against the peripheral and free ending nerves of the skin. Usually reactivity to peripheral nerves can be seen in patients with diabetes and/or peripheral neuropathies, but not with as much immune response to the free epidermal fibers as seen in our case. We believe that our case may represent an allergic drug reaction, overlapping with a possible environmental exposure to a neurotoxic agent.

In the same period as we evaluated this case, our laboratory noted several patients from rural areas of Georgia that exhibited similar neuropathologic features as this patient. We thus reported our series of patients from rural Georgia with both skin blisters and neural reactivity to the US Centers for Disease Control (CDC) in Atlanta, because these findings could occur simultaneously with environmental contamination with ricin, or other neurotoxins [8,9]. Such a possibility is suggested when classical known disease patterns do not fit observed neuropathologic data over multiple patients.

Ricin represents a significant potential biological toxin because of its stability and worldwide availability as a byproduct of castor oil production. In addition, it may be a potential agent of bioterror $[8,9]$.

We conclude that many conditions can cause blistering diseases; in selected cases, medication reactions and immunologic responses to neurotoxic agents may contribute to overall disease pathophysiology.

\section{REFERENCES}

1. Lipozenčić J, Marinović B: Bullous skin diseases. Clin Dermatol. 2011;29:353-4.

2. Park KY, Kim BJ, Kim MN: Amlodipine-associated bullous pemphigoid with erythema multiforme-like clinical features. Int $\mathrm{J}$ Dermatol. 2011;50:637-9.

3. Ibn Sellam A, Soualhi M, Zahraoui R, Marc K, Benamor J, Bourkadi JE, et al: [A rare form of rifampicin-induced skin toxicity: bullous pemphigoid]. Rev Mal Respir. 2011;28:365-71.

4. Abreu Velez AM, Klein AD, Howard MS: Bullous allergic drug eruption with presence of myeloperoxidase and reorganization of the dermal vessels observed by using CD34 and collagen IV antibodies. North Am J Med Sci. 2011;3:82-4.

5. Abreu Velez AM, Girard JG, Howard MS: IgG bullous pemphigoid with antibodies to $\operatorname{IgD}$, dermal blood vessels, eccrine glands and the endomysium of monkey esophagus. N Dermatol Online. 2011;2:48-51.

6. Bellón T, Blanca M: The innate immune system in delayed cutaneous allergic reactions to medications. Curr Opin Allergy Clin Immunol. 2011;11:292-8.

7. Foureur N, Descamps V, Lebrun-Vignes B, Picard-Dahan C, Grossin M, Belaich S, et al: Bullous pemphigoid in a leg affected with hemiparesia: a possible relation of neurological diseases with bullous pemphigoid? Eur J Dermatol. 2001;11:230-3.

8. Balint GA: Ricin: the toxic protein of castor oil seeds. Toxicology. 1974;2:77-102.

9.http://www.washingtonpost.com/blogs/checkpoint-washington/ post/ricin-plot-in-georgia-was-a-long-shot-experts-say/2011/11/02/ gIQAxBH6fM_blog.html. 


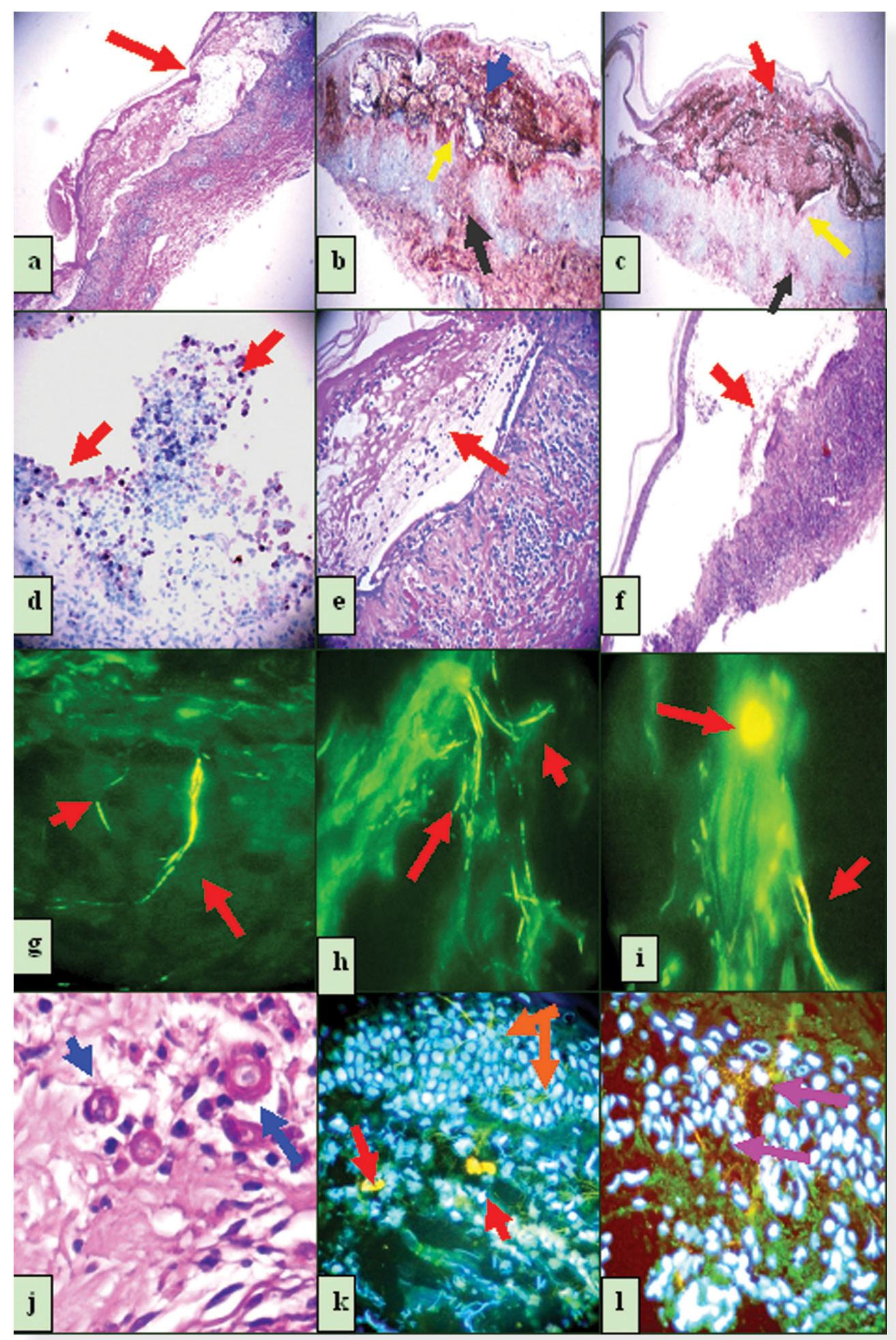

Figure 1. a. H\&E showing a subepidermal blister with an intraluminal inflammatory infiltrate (100X). b. IHC showing C3 staining inside the blister (brown staining; red arrow), in some areas of the BMZ (brown staining; yellow arrow), and in focal areas of the papillary dermis (brown staining; black arrow). c. IHC showing similar pattern of positivity as in $\mathbf{b}$, but utilizing anti-human albumin antibody. Note the staining inside the blister lumen (brown staining; red arrow), some staining in focal areas of the BMZ (brown staining; yellow arrow), and in the papillary dermis (brown staining; black arrow). d. IHC positive staining using antihuman IgE antibody on some cells inside the blister (dark brown staining; red arrows). e, f. H\&E at higher magnifications, highlighting the blister contents (red arrows). $\mathbf{g}$ and $\mathbf{h}$. DIF positive staining using FITC conjugated anti-human C1q against several neurovascular bundles in the dermis (yellow/green staining; red arrows). $\mathbf{i}$. Similar to $\mathbf{g}$ and $\mathbf{h}$, but at higher magnification (400X). j. PAS positive staining around the dermal neurovascular packages(blue arrows). k. Anti-human kappa antibody FITC conjugated showing positive staining in the neurovascular packages of the upper dermis (yellow staining; red arrows), please notice some thin fibers in the epidermis (green-yellow staining; orange arrow). 1. Additional positive staining of thin neural fibers in the epidermis, but in this case using FITC conjugated anti-human IgG (green staining; fuchsia arrows). 


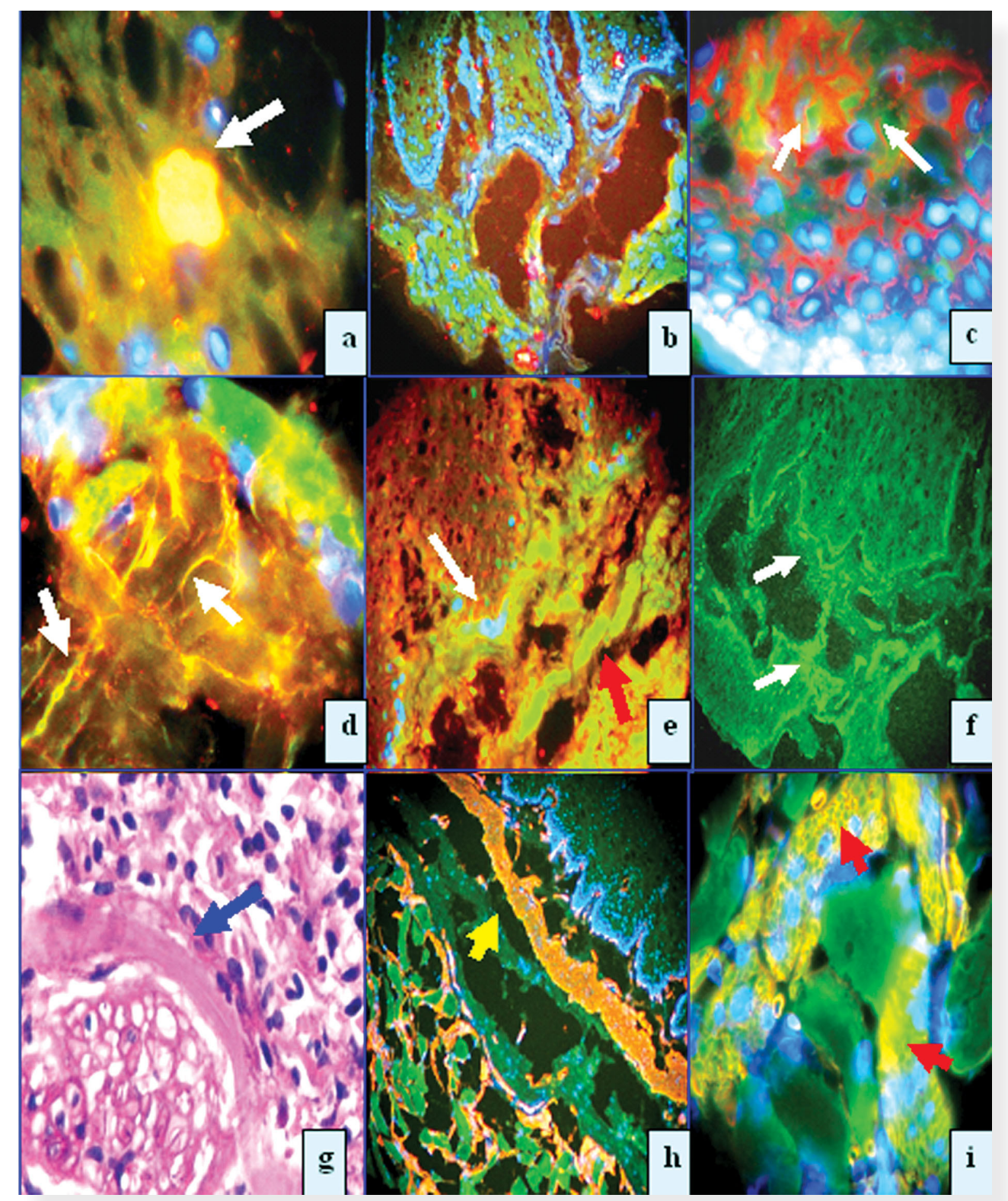

Figure 2. a. Double IIF staining utilizing FITC conjugated anti-human IgG antibody and Texas red conjugated anti-ARVCF antibody on monkey esophagus, and showing positive staining of a neural structure (flower-shaped structure; orange-yellow staining, white arrow)(100X). b. Double staining IIF on ME, showing positive stain with FITC conjugated anti-human kappa antibody (green staining; white arrow) and Texas red conjugated PPG 9.5 antibody(red staining) showing positivity in neural structures in the epithelium and BMZ (white arrows). c. IIF positive staining of neural structures on ME using FITC conjugated anti-human C4, and showing positive nerves in the epithelium (green staining, white arrows). The red staining between the cells is Texas red conjugated p0071 (100x). The nuclei of the epithelial keratinocytes are counterstained with Dapi (light blue staining). d. IIF on ME, demonstrating positive staining with FITC conjugated anti-human-C1q against several neural structures in the BMZ and beneath it (yellow-green staining; white arrows). e. IIF on ME, demonstrating positive staining with FITC conjugated anti-human-IgG against a neurovascular plexus in the epithelium (green staining; white arrow), and in the endomysium (green staining; red arrow). We also used Texas red conjugated armadillo repeat gene deleted in velocardiofacial syndrome (ARVCF) antibody, showing staining between the epithelial cells of the monkey esophagus (red staining). f. Similar to e, but using only the FITC conjugated anti-human IgG antibody (green staining; white arrows). g. PAS stain, showing some perineural staining and indicating the immune response occurs at this level (blue arrow; 400X). h. IIF on ME. Here we used Texas red conjugated anti-collagen IV antibody to address whether neural fibers crossed the endomysium collagen fibers (orange staining; yellow arrow) and the BMZ (blue staining). We were able to confirm that, as showing in higher magnification in $\mathbf{i}$ using a FITC conjugated anti-human kappa antibody, that nerves indeed tangentially cross the endomysium (yellow staining; red arrows).

Copyright by Ana Maria Abreu Velez et al. This is an open access article distributed under the terms of the Creative Commons Attribution License, which permits unrestricted use, distribution, and reproduction in any medium, provided the original author and source are credited. 